\title{
APSIDAL ANGLES FOR SYMMETRICAL DYNAMICAL SYSTEMS WITH TWO DEGREES OF FREEDOM
}

\author{
J. L. SYNGE
}

The bob of a spherical pendulum (or particle on a smooth sphere under gravity) oscillates between two levels, and the change of azimuth in passing from the lower to the higher level, or vice versa, is the same for all such passages during a single motion. Using the language of orbit theory, we shall refer to this change of azimuth as the apsidal angle. It is a function of the two constants of the motion, total energy and angular momentum, but the remarkable fact is that the apsidal angle $\alpha$ always satisfies the inequalities ${ }^{1}$

$$
\pi / 2<\alpha<\pi \text {. }
$$

The purpose of the present note is not to add to the theory of the spherical pendulum, but rather to place the problem in a more general setting. Consider a particle of unit mass which moves on a surface of revolution $\Sigma$ under the action of a conservative field of force for which the potential energy is independent of azimuth.

Let $A$ be the axis of $\Sigma$. Then $\Sigma$ is determined completely by the section $C$ of $\Sigma$ by a half-plane terminated by $A$. Since the particle cannot pass from a portion of $\Sigma$ to a disconnected portion of $\Sigma$, we may suppose $C$ to be a single connected curve.

Several cases present themselves:

(i) $C$ is an open curve with both ends on $A$ ( $\Sigma$ homeomorphic to a sphere).

(ii) $C$ is an open curve with one end on $A$ and the other end at infinity ( $\Sigma$ homeomorphic to a paraboloid of revolution).

(iii) $C$ is an open curve with both ends at infinity ( $\Sigma$ homeomorphic to a cylinder).

(iv) $C$ is a closed curve which does not meet $A$ ( $\Sigma$ homeomorphic to a torus).

In any one of these cases, we assign coordinates $R, \phi$, where $R$ is arc length measured along $C$ and $\phi$ is the azimuthal angle. The range of $\phi$ is taken to be $(-\infty, \infty)$, an increase of $2 \pi$ in $\phi$ leading us back to the same point. The range of $R$ depends on the type of $C$. In case (i), the range of $R$ is finite; in case (ii), the range may be taken as

Presented to the Society, April 26, 1947; received by the editors April 12, 1947.

${ }^{1}$ For references to earlier work, see $\mathrm{P}$. Appell, Traité de mécanique rationnelle, vol. 1 (1926) p. 518; see also A. Weinstein, Amer. Math. Monthly vol. 49 (1942) pp. 521-523, W. Kohn, Trans. Amer. Math. Soc. vol. 59 (1946) pp. 107-131. 
$(0, \infty)$; in case (iii), the range is $(-\infty, \infty)$; in case (iv), the range is taken to be $(-\infty, \infty)$, an increase in $R$ equal to the length of $C$ leading us back to the same point.

The line element of $\Sigma$ may be written

$$
d \sigma^{2}=d R^{2}+B(R) d \phi^{2},
$$

and the potential energy is $V(R)$. At any point common to $\Sigma$ and the axis $A$ we have $B(R)=0$. In case (iv) $B$ and $V$ are periodic functions of $R$, the period being the length of $C$. We shall assume $B$ and $V$ to be of class $C_{2}$ in all domains where the motion of the particle is considered.

Although the dynamical system described above consists of a particle on a surface of revolution, it is clear that any results obtained will be available also for a conservative dynamical system with two degrees of freedom and one ignorable coordinate, the Lagrangian function being

$$
L=2^{-1}\left[A(R) \dot{R}^{2}+B(R) \dot{\phi}^{2}\right]-V(R) .
$$

We shall, however, continue to refer to the system as if it consisted of a particle on a surface of revolution.

According to Jacobi's principle of stationary action, the path of the particle, if moving with total energy $E$, is a geodesic in a two-space with metric $d s$ where

$$
d s^{2}=(E-V) d \sigma^{2} .
$$

Let us introduce instead of $R$ a new coordinate $r$, defined by

$$
r=\int^{R}[E-V(u)]^{1 / 2} d u,
$$

the lower limit of integration being arbitrary. Then (4) becomes, by use of (2) and (5),

$$
d s^{2}=d r^{2}+G(r) d \phi^{2}
$$

where

$$
G(r)=[E-V(R)] B(R) .
$$

The form (6) may itself be regarded as the line element of a surface of revolution S. Accordingly the study of particle paths on a surface of revolution $\Sigma$ reduces to the study of geodesics on another surface of revolution $S .^{2}$

${ }^{2}$ In general, $S$ cannot be imbedded in a Euclidean 3-space. Since we shall be concerned solely with the intrinsic properties of $S$, that fact is not important for the purposes of the present paper. 
The geodesics for (6) satisfy the Lagrangian equations

$$
\frac{d}{d s} \frac{\partial w}{\partial r^{\prime}}-\frac{\partial w}{\partial r}=0, \quad \frac{d}{d s} \frac{\partial w}{\partial \phi^{\prime}}-\frac{\partial w}{\partial \phi}=0,
$$

where the prime means $d / d s$ and

$$
w=2^{-1}\left[\boldsymbol{r}^{\prime 2}+G(\boldsymbol{r}) \boldsymbol{\phi}^{\prime 2}\right] .
$$

Since $\phi$ is ignorable, we have the first integral

$$
G(r) \phi^{\prime}=h,
$$

a constant analogous to the constant of angular momentum. Equation (10) replaces the second of (8); instead of the first of (8), we may use the first integral

$$
\boldsymbol{r}^{\prime 2}+G(\boldsymbol{r}) \boldsymbol{\phi}^{\prime 2}=1 .
$$

Elimination of $s$ between (10) and (11) gives

$$
(d r / d \phi)^{2}=G\left(G-h^{2}\right) / h^{2} .
$$

The two constants, $E$ and $h$, are involved in the theory, but they play very different roles. The constant $E$ is implicit in the line element (6), through $G$, but the line element does not involve $h$. Since

$$
E=T+V,
$$

where $T$ is the kinetic energy, and $T$ cannot be negative, we see that $E$ is greater than or equal to $V$ at any point in the path of the particle. This places on $E$ the condition

$$
E \geqq \text { minimum of } V \text { on } \Sigma \text {. }
$$

The constant $h$ must satisfy a more exacting inequality. Since $r^{\prime 2} \geqq 0$ and $G(r) \geqq 0$ by (7) and the positive definite character of (2),

$$
h^{2} \leqq G(r)
$$

follows from (10) and (11), for all values of $r$ on the particle path; this may also be seen directly from (12).

The apsides of a particle path correspond to maximum and minimum values of $R$, and the apsidal angle $\alpha$ is the increment in the azimuthal angle $\phi$ in passing from minimum to maximum or from maximum to minimum. ${ }^{3}$ Since the transformation (5) is monotone,

${ }^{3}$ The surface of revolution is a particular case of the radial manifold, and radial apsides coincide with potential apsides; cf. J. L. Synge, Trans. Amer. Math. Soc. vol. 34 (1932) pp. 481-522. 
and $\phi$ is not transformed in the passage from $\Sigma$ to $S$, it follows that the apsidal distances $\left(R_{1}, R_{2}\right)$ for the particle path on $\Sigma$ correspond by (5) to the apsidal distances $\left(r_{1}, r_{2}\right)$ for the corresponding geodesic on $S$, and the apsidal angles are equal.

To find the apsidal distances, we put $d r / d \phi=0$ in (12). We have then to consider the two equations $G=0$ and $G-h^{2}=0$. By (10), $G=0$ implies $h=0$, and so $G=0$ implies $G-h^{2}=0$. Consequently we have to consider only the equation

$$
G=h^{2} \text {. }
$$

However, the case $h=0$ is singular, and we shall exclude it from further consideration. By (10), it implies $\phi^{\prime}=0$ for values of $r$ not making $G=0$, and so the motion is along a meridian curve (simple pendulum motion for a spherical pendulum).

If (16) has no roots, there are no apsides. To study the existence of apsides, let us regard $E$ as assigned, so that the function $G(r)$ is determined. Oscillation between apsidal distances $r_{1}, r_{2}\left(r_{1}<r_{2}\right)$ will occur if, and only if, there exists a pair of roots of (16):

$$
G\left(r_{1}\right)=h^{2}, \quad G\left(r_{2}\right)=h^{2} \quad\left(r_{1}<r_{2}\right),
$$

with the inequality (15) satisfied for all intermediate values of $r$ :

$$
G(r) \geqq h^{2} \quad\left(r_{1} \leqq r \leqq r_{2}\right) .
$$

The scheme for finding apsidal distances is then clear. The constant $E$ being assigned, we consider the graph of $G(r)$ versus $r$. Unless the graph shows at least one relative maximum, a pair of apsidal distances cannot occur. Let us suppose that $r=r_{0}$ gives a relative maximum, so that the derivative vanishes:

$$
(d G / d r)_{r=r_{0}}=0 .
$$

We then draw a parallel to the $r$-axis below this maximum, cutting the curve of $G(r)$ at $r_{1}$ and at $r_{2}$, with $r_{1}<r_{0}<r_{2}$, and assign to $h$ the value given by (17). Then there exists a geodesic with apsidal distances $r_{1}, r_{2}$, and, by (12), the apsidal angle is given by

$$
\alpha=\int_{r=r_{1}}^{r-r_{2}} d \phi=h \int_{r_{1}}^{r_{2}}\left[G\left(G-h^{2}\right)\right]^{-1 / 2} d r \text {. }
$$

It is interesting to note that if we had chosen $h^{2}=G\left(r_{0}\right)$ we would have obtained a circular geodesic (corresponding to the conical pendulum). This is easily seen from equations (8)-(12). In fact, any geodesic on $S$ possessing two apsidal distances weaves in and out across a circular geodesic. 
Let us now consider bounds for the apsidal angle $\alpha$, assuming that in the range $\left(r_{1}, r_{2}\right)$ there is only one value $r_{0}$ satisfying (19). Then in each of the ranges $\left(r_{1}, r_{0}\right),\left(r_{0}, r_{2}\right) G$ is a monotone function of $r$. We may write (20) in the form

$$
\begin{aligned}
\alpha= & h \int_{r=r_{1}}^{r=r_{0}}\left[G\left(G-h^{2}\right)\right]^{-1 / 2}|d r / d G| d G \\
& +h \int_{r=r_{2}}^{r=r_{0}}\left[G\left(G-h^{2}\right)\right]^{-1 / 2}|d r / d G| d G \\
= & h \int_{h^{2}}^{G_{0}}\left[G\left(G-h^{2}\right)\right]^{-1 / 2}\left[|d r / d G|_{1}+|d r / d G|_{2}\right] d G,
\end{aligned}
$$

where $G_{0}=G\left(r_{0}\right)$, and the subscripts 1 and 2 refer respectively to the ranges $\left(r_{1}, r_{0}\right)$ and $\left(r_{0}, r_{2}\right)$.

Consider now the ratio

$$
\frac{|d G / d r|}{\left(G_{0}-G\right)^{1 / 2}}
$$

in the range $\left(r_{1}, r_{2}\right)$. As $r$ approaches $r_{0}$, this ratio has the limit

$$
\left(-2 d^{2} G / d r^{2}\right)^{1 / 2} \text {, }
$$

evaluated at $r=r_{0}$. Accordingly the ratio (22) is bounded above and below, and we may write

$$
m \leqq\left(G_{0}-G\right)^{-1 / 2}|d G / d r| \leqq M \quad\left(r_{1} \leqq r \leqq r_{2}\right),
$$

where $m$ and $M$ are in general functions of $E$ and $h$. Equivalently,

$$
M^{-1}\left(G_{0}-G\right)^{-1 / 2} \leqq|d r / d G| \leqq m^{-1}\left(G_{0}-G\right)^{-1 / 2} \quad\left(r_{1} \leqq r \leqq r_{2}\right) .
$$

We might have $m=0$, in which case the upper bound is infinite. With the inequalities (24), equation (21) gives

$$
M^{-1} I \leqq \alpha \leqq m^{-1} I
$$

where

$$
I=2 h \int_{h^{2}}^{G_{0}}\left[G\left(G_{0}-G\right)\left(G-h^{2}\right)\right]^{-1 / 2} d G
$$

Changing the variable of integration by the transformation

$$
G=h^{2} \cos ^{2} \theta+G_{0} \sin ^{2} \theta,
$$

we get 


$$
I=4 \int_{0}^{\pi / 2}\left[1+\left(G_{0} / h^{2}-1\right) \sin ^{2} \theta\right]^{-1 / 2} d \theta
$$

and hence, since $G_{0}>h^{2}$,

$$
2 \pi h G_{0}^{-1 / 2}<I<2 \pi .
$$

Substitution in (25) gives the following result: For motion on a surface of revolution $\Sigma$ (or, equivalently, for a geodesic on a surface of revolution $S$ with line element (6)), the apsidal angle $\alpha$ satisfies the inequalities

$$
2 \pi M^{-1} h G_{0}^{-1 / 2}<\alpha<2 \pi m^{-1},
$$

where $h$ is the constant of the first integral (10), $G_{0}$ is $G\left(r_{0}\right)$ where $r_{0}$ satisfies (19), and $M, m$ are respectively upper and lower bounds of the ratio (22) in the range of oscillation $\left(r_{1}, r_{2}\right)$, determined by $G=h^{2}$.

It is interesting to see what happens when the geodesic under consideration lies close to the circular geodesic $r=r_{0}$. Then $h^{2}$ approaches $G_{0}$ and the two bounds $M$ and $m$ approach the common value $\left(-2 d^{2} G / d r^{2}\right)^{-1 / 2}$ evaluated at $r_{0}$; we get in the limit

$$
\alpha=2 \pi\left(-2 d^{2} G / d r^{2}\right)^{-1 / 2},
$$

evaluated at $r_{0}$. This value is easily checked by differentiating (12), dividing by $d r / d \phi$, and linearizing the resulting equation.

Having transformed the problem of the apsidal angle for motion on a surface of revolution to that for geodesics on a surface of revolution, we can bring to bear on it well known results in differential geometry. Consider, on the surface of revolution $S$, the triangle $A O B$ the sides of which are geodesics. Let $O$ be the pole of $S$, that is, the point where it is met by the axis. Then $O A$ and $O B$ are meridians. Further, suppose that $A$ and $B$ are adjacent apsides on the geodesic $A B$, so that the angles $A B O$ and $B A O$ are right angles and $A O B$ is the apsidal angle $\alpha$. Then $\alpha$ is the excess of the angle sum over $\pi$, and so by the well known formula

$$
\alpha=\iint K d S,
$$

where $K$ is the Gaussian curvature of $S$ for the metric (6) and the integration extends over the triangle $A O B, d S$ being the element of area.

The formula (32) brings an interesting fact to light. Consider the small oscillations of a spherical pendulum about the position of equilibrium. It is known that the particle path is approximately a 
central ellipse $(\alpha=\pi / 2)$. Consider the triangle $A O B$ as described above. To that triangle on $S$ there corresponds on the sphere $\Sigma$ a triangle which we may also call $A O B$; the sides $A O$ and $O B$ are great circles joining $O$, the lowest point of the sphere $\Sigma$, to adjacent apsides $A$ and $B ; A B$ is the particle path between apsides.

Now, for small oscillations, the area of the triangle $A O B$ on $\Sigma$ is small. The area of $A O B$ on $S$ is still smaller, since the factor $E-V$ involved in passing from $d \sigma^{2}$ to $d s^{2}$ is small for small kinetic energy. How then can the integral in (32) have a finite value, approximately $\pi / 2$ ? The answer is to be found in $K$. For the metric (6), the circle $V=E$ is singular, and the Gaussian curvature of $S$ tends to infinity as we approach this circle. If we take the zero of potential energy at the lowest point of the sphere, that is, at the point $O$, then small oscillations correspond to small values of $E$, and the radius of the circle $V=E$ is small. But inside this circle, considered in $S$, the Gaussian curvature is great, in such a way that its greatness balances the smallness of the area of the circle.

These considerations point to the desirability of the further investigation of the geometry of dynamical systems when the Jacobi metric (6) is used; this remark applies not merely to the simple symmetric case discussed, above, but to a general dynamical system. The locus $V=E$ has an interesting geometry. It is a null-domain, in the sense that the length of any curve drawn in it is zero. It forms a barrier across which a geodesic cannot pass; in fact, geodesics approaching it are bent back sharply. Furthermore, on it the curvature becomes infinite.

It might seem that the reduction of the question of dynamical apsidal angles to the question of geodesic apsidal angles might yield some simple general bounds. So we ask: For the whole class of surfaces of revolution, do there exist upper and lower bounds for apsidal angles? We shall show that the answer is in the negative, by constructing models in which the apsidal angles pass any given bound. It is simplest to use limiting models with sharp edges; passage to the limit from smooth surfaces will not destroy the inequalities.

Consider a long circular cylinder with flat ends, the pole $O$ being the center of one end $E$. Consider a geodesic which consists in part of a chord of $E$, not a diameter. The midpoint of this chord is an apse; in fact, it is a point of minimum distance from $O$. Pursuing the geodesic in one direction, it passes over the edge of $E$, and winds round and round the curved sides of the cylinder. The azimuthal angle increases by $2 \pi$ in each such revolution, and the next apse cannot occur until the geodesic reaches the other end of the cylinder. 
Obviously, by making the cylinder sufficiently long, we can make the apsidal angle as great as we please.

To get a small apsidal angle, we take as our surface the two faces of a flat circular disk. The pole $O$ is the center of one face $E$. Consider a geodesic consisting in part of a chord of $E$ which subtends at $O$ a small angle $\beta$. The midpoint of the chord is an apse. Follow this geodesic in one direction over the edge of the disk and on to the other face $E^{\prime}$. The chord on $E^{\prime}$ and the chord on $E$ will make equal angles with the radius drawn from $O$ to the point where the geodesic passes over the edge. The midpoint of the chord on $E^{\prime}$ will also be an apse. The apsidal angle is $\alpha=\beta$, and can be made as small as we please by making $\beta$ sufficiently small.

Consequently, for the whole class of surfaces of revolution, there is neither an upper nor a lower bound for the geodesic apsidal angle.

Carnegie Institute of Technology 